\title{
Sertraline maintenance treatment reduced relapse and dropouts in post-traumatic stress disorder
}

Davidson J, Pearlstein T,Londborg P, et al. Efficacy of sertraline in preventing relapse of posttraumatic stress disorder: results of a 28- week double-blind, placebo-controlled study. Am J Psychiatry 2001 Dec;158:1974-81.

\section{QUESTION: In patients who have post-traumatic stress disorder (PTSD) and have responded to continuation sertraline treatment, does maintenance sertraline treatment reduce relapse?}

\section{Design}

Randomised (unclear allocation concealment*), blinded (unclear)*, placebo controlled trial with 28 weeks of follow up.

\section{Setting}

24 centres in the US.

\section{Patients}

96 patients who were 21-69 years of age (mean age $43 \mathrm{y}$, $70 \%$ women); met the DSM-III-R criteria for PTSD; had PTSD symptoms for $>6$ months (mean duration $13 \mathrm{y}$ ) and a total severity score $\geq 50$ on the ClinicianAdministered PTSD Scale (CAPS) part 2; and met responder criteria (Clinical Global Impression [CGI] improvement score $\leq 2$ and $\geq 30 \%$ improvement in total severity score on CAPS part 2) after 24 weeks of sertraline. Exclusion criteria included bipolar disorder; schizophrenia; organic mental disorder; primary diagnosis of major depression or anxiety; and substance abuse. Follow up was $88 \%$.

\section{Intervention}

Patients were allocated to sertraline, $50-200 \mathrm{mg} / \mathrm{day}$ (mean dose $137 \mathrm{mg} / \mathrm{d}) \quad(\mathrm{n}=46)$ or placebo $(\mathrm{n}=50)$. Concomitant psychotropic therapy (except for chloral hydrate on $\leq 2$ nights/wk) or cognitive behaviour therapy was not permitted. Other forms of psychotherapy could not begin or end during the study period.

\section{Main outcome measures}

Relapse, dropout because of clinical deterioration, acute exacerbation, study completers, and adverse events. Patients who relapsed met 3 criteria on 2 consecutive visits: CGI improvement score $\geq 3 ; \geq 30 \%$ increase and increase of $\geq 15$ points on CAPS, part 2; and substantial worsening of patients' clinical condition as judged by the investigator.

\section{Main results}

Analysis was by intention to treat. Sertraline led to lower rates of relapse, non-completion of study, and acute exacerbation than did placebo (table). No adverse events with a rate $\geq 10 \%$ occurred in the sertraline group; dizziness was the only adverse event with a rate $\geq 10 \%$ in the placebo group $(4.3 \%$ for sertraline $v 18 \%$ for placebo, $\mathrm{p}=0.05$ ).

A

this abstract and

commentary appears in

Evidence-Based

Medicine.

Sertraline v placebo for sertraline responders with post-traumatic stress disorder

\begin{tabular}{|c|c|c|c|c|}
\hline Outcomes at 28 weeks & Sertraline & Placebo & RRR $(95 \%$ Cl) & NNT (Cl) \\
\hline Relapse & $5.3 \%$ & $26 \%$ & $80 \%$ (27 to 95$)$ & 5 (3 to 19$)$ \\
\hline $\begin{array}{l}\text { Relapse or discontinuatio } \\
\text { because of clinical } \\
\text { deterioration }\end{array}$ & $16 \%$ & $46 \%$ & $65 \%$ (27 to 85$)$ & $3(2$ to 10$)$ \\
\hline Acute exacerbation & $16 \%$ & $52 \%$ & $70 \%$ (37 to 86$)$ & 3 (2 to 7$)$ \\
\hline Did not complete study & $39 \%$ & $60 \%$ & $35 \%(1.7$ to 58$)$ & 5 (3 to 135$)$ \\
\hline
\end{tabular}

\section{Conclusion}

In patients who had post-traumatic stress disorder and had responded to sertraline, maintenance sertraline reduced relapse and dropouts.

*See glossary.

\section{COMMENTARY}

Davidson et al report the first published randomised controlled trial (RCT) of maintenance treatment for PTSD. The idea of applying the standard treatment for depression to PTSD by continuing treatment with an antidepressant for 6 months after response ${ }^{1}$ appears to be challenged.

This RCT is of far higher quality than many previous PTSD treatment studies. However, several methodological issues call for cautious interpretation of the results. The patients represent only a small proportion of those who entered the research programme. All patients completed a 12 week acute phase RCT comparing sertraline with placebo plus a 24 week open label sertraline continuation study. Of the initial 380 patients who entered the acute phase trial, 155 completed the open label trial. Davidson et al then randomised 96 of these patients in the current trial with only 48 completers.

Twice as many men were in the placebo group as in the sertraline group. This difference is of concern because none of the 9 men in the sertraline group relapsed, whereas 5 of the 18 men in the placebo group did. Another potential issue is that 38 patients $(40 \%)$ met criteria for a secondary depressive disorder. The authors did not state how many of these patients were in each group, but stated that the presence of depressive disorder did not influence relapse rates. Perhaps of more importance is the failure to report how many patients were originally randomised to sertraline in the acute phase RCT. The result is that some patients will have already taken sertraline for 36 weeks by study entry and others for 24 weeks.

Notwithstanding these issues, the results convincingly favour sertraline maintenance. Most relapses occurred between 3 and 8 weeks after stopping sertraline, supporting the authors' conclusion that loss of prophylactic efficacy was more likely to explain the difference than a discontinuation syndrome. If these results prove correct it could be strongly argued that patients with chronic PTSD who respond to sertraline should not have it discontinued within a year, particularly because it appears to be well tolerated. Future research may identify subgroups that are more likely to need longer maintenance treatment than others. In this study, patients who responded most quickly in the acute phase trial were those least likely to relapse on discontinuation. Jonathan I Bisson, DM, MRCPsych University Hospital of Wales Cardiff, $U K$

1 Loonen AJ, Peer PG, Zwanikken GJ. Continuation and maintenance therapy with antidepressive agents. Meta-analysis of
research. Pharm Weekbl Sci 1991;13:167-75. 